PROCEEDINGS OF THE

AMERICAN MATHEMATICAL SOCIETY

Volume 133, Number 1, Pages 285-293

S 0002-9939(04)07528-8

Article electronically published on May 20, 2004

\title{
FOURIER TRANSFORMS OF STATIONARY PROCESSES
}

\author{
WEI BIAO WU
}

(Communicated by Richard C. Bradley)

\begin{abstract}
We consider the asymptotic behavior of Fourier transforms of stationary and ergodic sequences. Under sufficiently mild conditions, central limit theorems are established for almost all frequencies as well as for a given frequency. Applications to the widely used linear processes and iterated random functions are discussed. Our results shed new light on the foundation of spectral analysis in that the asymptotic distribution of the periodogram, the fundamental quantity in the frequency-domain analysis, is obtained.
\end{abstract}

\section{INTRODUCTION}

Let $\left(X_{n}\right)_{n \in \mathbb{Z}}$ be a stationary and ergodic Markov chain on the state space $\mathcal{X}$; let $g$ be a real-valued function on $\mathcal{X}$ such that $\mathbb{E}\left[g\left(X_{0}\right)\right]=0$ and $\mathbb{E}\left[\left|g^{2}\left(X_{0}\right)\right|\right]<\infty$. Define the Fourier transform

$$
S_{n}(\theta)=S_{n}(\theta ; g)=\sum_{k=1}^{n} g\left(X_{k}\right) e^{i k \theta}, \quad \theta \in \mathbb{R},
$$

where $i=\sqrt{-1}$ is the imaginary unit. The setup (1) is general enough to allow one to consider $S_{n}^{Y}(\theta)=\sum_{k=1}^{n} Y_{k} e^{i k \theta}$ for any stationary and ergodic process $\left(Y_{n}\right)_{n \in \mathbb{Z}}$ by constructing a Markov chain $X_{n}=\left(\ldots, Y_{n-1}, Y_{n}\right)$ and $g\left(X_{n}\right)=Y_{n}$. The quantity (1) is of fundamental importance in the spectral analysis of stationary processes.

This paper considers the asymptotic normality of (1). This problem has a substantial history. Rosenblatt (14, Theorem 5.3, p. 131) considers mixing processes; Brockwell and Davis (3], Theorem 10.3.2., p. 347), Walker [18] and Terrin and Hurvich [16] discuss linear processes. Other contributions can be found in Olshen [1], Rootzén [12, Yajima [23] and Walker [19] among others.

Under surprisingly weak conditions (cf. (2)), we show that the real and imaginary parts of $S_{n}(\theta) / \sqrt{n}$ are asymptotically iid normal for almost all frequencies $\theta \in \mathbb{R}$. Thus the periodogram $n^{-1}\left|S_{n}(\theta)\right|^{2}$ is asymptotically distributed as a multiple of a $\chi^{2}(2)$ random variable. For a fixed $\theta$, we present sufficient conditions for the asymptotic normality of $S_{n}(\theta) / \sqrt{n}$. Our general results go beyond earlier ones by providing mild and verifiable conditions. In our proof, we apply the

Received by the editors March 24, 2003 and, in revised form, June 27, 2003 and September 18, 2003.

2000 Mathematics Subject Classification. Primary 60F05, 60F17; Secondary 60G35.

Key words and phrases. Spectral analysis, linear process, martingale central limit theorem, periodogram, Fourier transformation, nonlinear time series.

(C)2004 American Mathematical Society 
celebrated Carleson theorem in Fourier analysis to construct approximating martingales. Applications to special sequences such as linear processes and iterated random functions, which are widely used in time series modelling, are discussed.

\section{MAIN RESULTS}

Let $(\Omega, \mathcal{B}(\Omega), \mathbb{P})$ be the probability space on which the sequence $X_{n}$ is defined, where $\mathcal{B}(\Omega)$ is a sigma algebra on $\Omega$. For a random variable $\xi$ on the space $(\Omega, \mathcal{B}(\Omega), \mathbb{P})$, we denote its $\mathcal{L}^{2}(\mathbb{P})$ norm by $\|\xi\|=\sqrt{\mathbb{E}\left(|\xi|^{2}\right)} \leq \infty$. Define the projection operator

$$
\mathcal{P}_{k} \xi=\mathbb{E}\left(\xi \mid \ldots, X_{k-1}, X_{k}\right)-\mathbb{E}\left(\xi \mid \ldots, X_{k-1}\right) .
$$

Then by the Markovian property, $\mathcal{P}_{1} g\left(X_{n}\right)=\mathbb{E}\left[g\left(X_{n}\right) \mid X_{1}\right]-\mathbb{E}\left[g\left(X_{n}\right) \mid X_{0}\right]$ for $n \in \mathbb{N}$. Let $\Re z$ and $\Im z$ be the real and the imaginary parts of the complex number $z$, namely $z=\Re z+i \Im z$, and let $|z|=\sqrt{(\Re z)^{2}+(\Im z)^{2}}$. Denote by $N(\mu, \Sigma)$ the multivariate normal distribution with mean vector $\mu$ and covariance matrix $\Sigma$; denote by $\operatorname{Id}_{p}$ the $p \times p$ identity matrix. Theorems 1 and 2 concern asymptotic normality for almost all $\theta$ and for a given $\theta$, respectively.

Theorem 1. Assume that

$$
\sum_{n=1}^{\infty} \frac{1}{n}\left\|\mathbb{E}\left[g\left(X_{n}\right) \mid X_{0}\right]\right\|^{2}<\infty .
$$

Then (i) for almost all $\theta \in \mathbb{R}$ (Lebesgue), there exists $0 \leq \sigma(\theta)<\infty$ such that

$$
\left(\begin{array}{c}
\Re \\
\Im
\end{array}\right) \frac{S_{n}(\theta)}{\sqrt{n}} \Rightarrow N\left[0, \sigma^{2}(\theta) \operatorname{Id}_{2}\right] .
$$

(ii) Moreover, for almost all pairs $(\theta, \varphi)$ (Lebesgue), $S_{n}(\theta) / \sqrt{n}$ and $S_{n}(\varphi) / \sqrt{n}$ are asymptotically independent.

Theorem 2. For a given $\theta \in[0,2 \pi)$, suppose there exists $\xi\left(\theta, X_{0}, X_{1}\right) \in \mathcal{L}^{2}(\mathbb{P})$ such that

$$
\sum_{n=1}^{N} e^{i n \theta} \mathcal{P}_{1} g\left(X_{n}\right) \rightarrow \xi\left(\theta, X_{0}, X_{1}\right) \quad\left(\mathcal{L}^{2}(\mathbb{P})\right)
$$

as $N \rightarrow \infty$, and

$$
\left\|\mathbb{E}\left[S_{n}(\theta) \mid X_{0}\right]\right\|^{2}=o(n) .
$$

Then (i) if $\theta \neq 0, \pi$,

$$
\left(\begin{array}{c}
\Re \\
\Im
\end{array}\right) \frac{S_{n}(\theta)}{\sqrt{n}} \Rightarrow N\left[0, \sigma^{2}(\theta) \operatorname{Id}_{2}\right],
$$

where $\sigma^{2}(\theta)=\left\|\xi\left(\theta, X_{0}, X_{1}\right)\right\|^{2} / 2$, and (ii) $S_{n}(\theta) / \sqrt{n} \Rightarrow N\left(0, \sigma^{2}\right)$ if $\theta=0$ or $\pi$, where $\sigma^{2}(\theta)=\left\|\xi\left(\theta, X_{0}, X_{1}\right)\right\|^{2}$.

Proposition 1. Assume that

$$
\sum_{n=1}^{\infty}\left\|\mathcal{P}_{1} g\left(X_{n}\right)-\mathcal{P}_{1} g\left(X_{n+1}\right)\right\|<\infty .
$$

Then (4) and (15), and consequently (6), hold for all $0<\theta<2 \pi$. 
Condition (2) is fairly mild and imposes a very weak decay rate of $\left\|\mathbb{E}\left[g\left(X_{n}\right) \mid X_{0}\right]\right\|$. Rootzén [12] obtained a central limit theorem under conditions which are not easily verifiable. In comparison, our conditions (44), (15) and (7) are tractable in many cases. It is easily seen that (2) is equivalent to

$$
\sum_{k=1}^{\infty} 2^{-k} \sum_{i=1}^{2^{k}}\left\|\mathbb{E}\left[g\left(X_{i}\right) \mid X_{0}\right]\right\|^{2}<\infty
$$

by exchanging the order of summation. Notice that $\left\|\mathbb{E}\left[g\left(X_{n}\right) \mid X_{0}\right]\right\|$ is non-increasing in $n$ in view of $\left\|\mathcal{P}_{-n} g\left(X_{0}\right)\right\|^{2}=\left\|\mathbb{E}\left[g\left(X_{0}\right) \mid X_{-n}\right]\right\|^{2}-\left\|\mathbb{E}\left[g\left(X_{0}\right) \mid X_{-n-1}\right]\right\|^{2}$. So another equivalent condition of (2) is $\sum_{k=1}^{\infty}\left\|\mathbb{E}\left[g\left(X_{2^{k}}\right) \mid X_{0}\right]\right\|^{2}<\infty$. Inequality (8) is needed to establish a connection between $\sigma(\theta)$ in (3) and spectral densities (cf. Proposition 21).

Let $r_{k}=\mathbb{E}\left[g\left(X_{0}\right) g\left(X_{k}\right)\right]$ be the covariance function and introduce the spectral distribution function $F(\theta), 0 \leq \theta \leq 2 \pi$, via Herglotz's Theorem,

$$
r_{k}=\int_{0}^{2 \pi} \exp (i k \theta) d F(\theta) \text {. }
$$

Assume that $F$ is absolutely continuous with the spectral density function $f$, namely $F(\theta)=\int_{0}^{\theta} f(u) d u$.

Proposition 2. Assume (21). Then for almost all $\theta \in[0,2 \pi]$ (Lebesgue), $f(\theta)=$ $\sigma^{2}(\theta) / \pi$.

Example 1. Iterated random functions. Let $(\mathcal{X}, \rho)$ be a complete and separable metric space and let $X_{n}=F_{\varepsilon_{n}}\left(X_{n-1}\right)$, where $F_{\varepsilon}(\cdot)=F(\cdot, \varepsilon)$ is the $\varepsilon$ section of a jointly measurable function $F: \mathcal{X} \times \Upsilon \mapsto \mathcal{X}$ and $\varepsilon, \varepsilon_{n}, n \in \mathbb{Z}$, are iid random variables that take values in a second measurable space $\Upsilon$. Define $L_{\varepsilon}=\sup _{x \neq x^{\prime}} \rho\left[F_{\varepsilon}(x), F_{\varepsilon}\left(x^{\prime}\right)\right] / \rho\left(x, x^{\prime}\right)$. Diaconis and Freedman [6] prove that $X_{n}$ admits a unique stationary distribution (say $\Pi$ ) if

$$
\mathbb{E}\left(L_{\varepsilon}^{\alpha}\right)<\infty, \mathbb{E}\left(\log L_{\varepsilon}\right)<0, \text { and } \mathbb{E}\left[\rho^{\alpha}\left(x_{0}, F_{\varepsilon}\left(x_{0}\right)\right)\right]<\infty
$$

for some $\alpha>0$ and $x_{0} \in \mathcal{X}$. Let $\Delta_{g}(\delta)=\sup \left\{\left\|\left[g(X)-g\left(X^{\prime}\right)\right] \mathbf{1}_{\left[\rho\left(X, X^{\prime}\right) \leq \delta\right]}\right\|\right.$ : $\left.X, X^{\prime} \sim \Pi\right\}$.

Corollary 1. Assume (9), $\mathbb{E}\left[g\left(X_{1}\right)\right]=0$ and $\mathbb{E}\left[\left|g\left(X_{1}\right)\right|^{\ell}\right]<\infty$ for some $\ell>2$. (a) If

$$
\int_{0}^{1 / 2} \frac{\Delta_{g}^{2}(t)}{t|\log t|} d t<\infty
$$

then (2) holds. (b) If

$$
\int_{0}^{1 / 2} \frac{\Delta_{g}(t)}{t} d t<\infty
$$

then $\sum_{n=1}^{\infty}\left\|\mathbb{E}\left[g\left(X_{n}\right) \mid X_{1}\right]\right\|<\infty$ and hence (7) holds.

Proof. Let $X_{0}^{\prime} \sim \Pi$, and let $X_{0}^{\prime}$ be independent of $X_{0}$ and $\left(\varepsilon_{k}\right)_{k \in \mathbb{Z}}$. For $n \geq 1$, let $X_{n}^{\prime}=F_{\varepsilon_{n}} \circ F_{\varepsilon_{n-1}} \circ \ldots \circ F_{\varepsilon_{1}}\left(X_{0}^{\prime}\right)$. Then (99) implies that there exists $\beta, C>0$ and $0<r<1$ such that $\mathbb{E}\left[\rho^{\beta}\left(X_{n}, X_{n}^{\prime}\right)\right] \leq C r^{n}$ hold for all $n \geq 0$ (cf. Lemma 3 in Wu 
and Woodroofe [22]). Let $p=\ell / 2, q=p /(p-1)$ and $\delta_{n}=\left(C r^{n}\right)^{1 /(q+\beta)}$. Since $\mathbb{E}\left[g\left(X_{n}^{\prime}\right) \mid X_{0}\right]=0$,

$$
\begin{aligned}
& \left\|\mathbb{E}\left[g\left(X_{n}\right) \mid X_{0}\right]\right\| \\
& \quad \leq\left\|\left[g\left(X_{n}\right)-g\left(X_{n}^{\prime}\right)\right] \mathbf{1}_{\rho\left(X_{n}, X_{n}^{\prime}\right)<\delta_{n}}\right\|+\left\|\left[g\left(X_{n}\right)-g\left(X_{n}^{\prime}\right)\right] \mathbf{1}_{\rho\left(X_{n}, X_{n}^{\prime}\right) \geq \delta_{n}}\right\| \\
& \quad \leq \Delta_{g}\left(\delta_{n}\right)+\left\|\left[g\left(X_{n}\right)-g\left(X_{n}^{\prime}\right)\right]^{2}\right\|_{p}^{1 / 2} \times\left[\mathbb{P}\left(\rho\left(X_{n}, X_{n}^{\prime}\right) \geq \delta_{n}\right)\right]^{\frac{1}{2 q}} \leq \Delta_{g}\left(\delta_{n}\right)+C^{\prime} \delta_{n}^{1 / 2} .
\end{aligned}
$$

So (21) follows since (10) entails $\sum_{n=1}^{\infty} \Delta_{g}^{2}\left(\delta_{n}\right) / n<\infty$. (b) similarly follows.

Example 2. Linear processes. Let $\varepsilon_{k}, k \in \mathbb{Z}$, be iid random variables with mean 0 and finite variance; let $X_{n}=\left(\ldots, \varepsilon_{n-1}, \varepsilon_{n}\right)$ and $g\left(X_{n}\right)=\sum_{i=0}^{\infty} a_{i} \varepsilon_{n-i}$, where $a_{i}$ are real numbers such that $\sum_{i=0}^{\infty} a_{i}^{2}<\infty$. Then (7) is reduced to $\sum_{i=1}^{\infty}\left|a_{i}-a_{i-1}\right|<$ $\infty$, and the central limit theorem (6) holds for all $0<\theta<2 \pi$. Notice that this cannot be extended to $\theta=0$ if $\left|a_{n}\right|$ is not summable. For example, $a_{n}=n^{-\beta}$, $1 / 2<\beta<1$ for $n \geq 1$.

\section{Proofs}

Let $\mathbb{U}$ be the uniform probability measure on $\Theta=[0,2 \pi)$, and let $\mathcal{B}(\Theta)$ be the class of the Borel sets of $\Theta$. Denote by $(\Theta \times \Omega, \mathcal{B}(\Theta) \times \mathcal{B}(\Omega), \mathbb{U} \times \mathbb{P})$ the product probability space of $(\Theta, \mathcal{B}(\Theta), \mathbb{U})$ and $(\Omega, \mathcal{B}(\Omega), \mathbb{P})$.

Lemma 1. Assume (21). Then for almost all $\theta \in \Theta(\mathbb{U})$,

$$
\frac{1}{\sqrt{n}} \mathbb{E}\left[S_{n}(\theta) \mid X_{0}\right] \rightarrow 0 \text { almost surely }(\mathbb{P}) .
$$

Proof of Lemma 1. Let $A \in \mathcal{B}(\Theta) \times \mathcal{B}(\Omega)$ be the set on which

$$
\sum_{n=1}^{N} \frac{1}{\sqrt{n}} e^{i n \theta} \mathbb{E}\left[g\left(X_{n}\right) \mid X_{0}\right]
$$

does not converge as $N \rightarrow \infty$. Let $A^{\omega}$ and $A_{\theta}$ be the $\omega$-section and $\theta$-section of $A$, respectively. Define

$$
\Omega_{0}=\left\{\omega \in \Omega: \sum_{n=1}^{\infty} \frac{1}{n}\left|\mathbb{E}\left[g\left(X_{n}\right) \mid X_{0}\right]\right|^{2}<\infty\right\} .
$$

By (2), $\mathbb{P}\left(\Omega_{0}\right)=1$. For $\omega \in \Omega_{0}$, by Carleson's theorem, $\mathbb{U}\left(A^{\omega}\right)=0$. Thus, by Fubini's theorem, for almost all $\theta \in \Theta(\mathbb{U}), \mathbb{P}\left(A_{\theta}\right)=0$, i.e., $\sum_{n=1}^{\infty} e^{i n \theta} \mathbb{E}\left[g\left(X_{n}\right) \mid X_{0}\right] / \sqrt{n}$ converges almost surely $(\mathbb{P})$. Hence by Kronecker's lemma (cf. Chow and Teicher [5], Lemma 5.1.2), as $N \rightarrow \infty$,

$$
\frac{1}{\sqrt{N}} \sum_{n=1}^{N} e^{i n \theta} \mathbb{E}\left[g\left(X_{n}\right) \mid X_{0}\right] \rightarrow 0 \quad \text { a.s. }
$$

Lemma 2. For almost all $\theta \in \Theta(\mathbb{U})$, there exists $\xi\left(\theta, X_{0}, X_{1}\right) \in \mathcal{L}^{2}$ such that

$$
\mathcal{P}_{1} S_{n}(\theta) \rightarrow \xi\left(\theta, X_{0}, X_{1}\right) \text { almost surely }(\mathbb{P}) .
$$


Proof of Lemma [. Since the sequence $\mathbb{E}\left[g\left(X_{0}\right) \mid X_{1-n}\right]-\mathbb{E}\left[g\left(X_{0}\right) \mid X_{-n}\right], n=0,1, \ldots$, forms martingale differences,

$$
\sum_{n=1}^{\infty}\left\|\mathcal{P}_{1} g\left(X_{n}\right)\right\|^{2}=\sum_{n=1}^{\infty}\left\|\mathcal{P}_{1-n} g\left(X_{0}\right)\right\|^{2}=\left\|g\left(X_{0}\right)\right\|^{2}<\infty .
$$

Hence for almost all $\omega \in \Omega(\mathbb{P}), \sum_{n=1}^{\infty}\left|\mathcal{P}_{1} g\left(X_{n}\right)\right|^{2}<\infty$, which yields (13) by Carleson's theorem and the same argument as in Lemma 1. Next we show that $\xi\left(\theta, X_{0}, X_{1}\right) \in \mathcal{L}^{2}$. To this end, since the trigonometric bases $e^{i n \cdot}, n \in \mathbb{Z}$, are orthogonal,

$$
\sum_{k=1}^{n}\left\|\mathcal{P}_{1-k} g\left(X_{0}\right)\right\|^{2}=\int_{\Theta} \mathbb{E}\left|\mathcal{P}_{1} S_{n}(\theta)\right|^{2} \mathbb{U}(d \theta) .
$$

By Fatou's lemma,

$$
\begin{aligned}
\left\|g\left(X_{0}\right)\right\|^{2} & \geq \liminf _{n \rightarrow \infty} \int_{\Theta} \mathbb{E}\left|\mathcal{P}_{1} S_{n}(\theta)\right|^{2} \mathbb{U}(d \theta) \\
& \geq \int_{\Theta} \mathbb{E} \liminf _{n \rightarrow \infty}\left|\mathcal{P}_{1} S_{n}(\theta)\right|^{2} \mathbb{U}(d \theta)=\int_{\Theta} \mathbb{E}\left[\left|\xi\left(\theta, X_{0}, X_{1}\right)\right|^{2}\right] \mathbb{U}(d \theta) .
\end{aligned}
$$

Hence for almost all $\theta \in \Theta(\mathbb{U}),\left\|\xi\left(\theta, X_{0}, X_{1}\right)\right\|^{2}<\infty$.

Lemma 3. Assume (2). Then for almost all $\theta \in \Theta(\mathbb{U})$,

$$
\mathbb{E}\left[\xi\left(\theta, X_{0}, X_{1}\right) \mid X_{0}\right]=0 \quad \text { a.s. }
$$

Proof of Lemma 3 . The almost sure convergence (13) in Lemma 2 alone does not guarantee the $\mathcal{L}^{2}$ convergence. To prove (14), we shall show below that, under (2), the Cesàro average $\sum_{n=1}^{N} \mathcal{P}_{1} S_{n}(\theta) / N$ converges to $\xi\left(\theta, X_{0}, X_{1}\right)$ in $\mathcal{L}^{2}$. The relation (14) reveals the martingale structure, and we can apply the martingale central limit theorem.

Let the tail

$$
R_{N}\left(\theta, X_{0}, X_{1}\right)=\sum_{n=N+1}^{\infty} e^{i n \theta}\left\{\mathbb{E}\left[g\left(X_{n}\right) \mid X_{1}\right]-\mathbb{E}\left[g\left(X_{n}\right) \mid X_{0}\right]\right\}
$$

Then $R_{0}\left(\theta, X_{0}, X_{1}\right)=\xi\left(\theta, X_{0}, X_{1}\right)$. Again by the orthogonality of the bases $e^{i n}$,

$$
\int_{\Theta}\left|R_{N}\left(\theta, X_{0}, X_{1}\right)\right|^{2} \mathbb{U}(d \theta)=\sum_{n=1+N}^{\infty}\left|\mathcal{P}_{1} g\left(X_{n}\right)\right|^{2}<\infty .
$$

Hence by (2),

$$
\begin{aligned}
\sum_{N=1}^{\infty} \frac{1}{N} \int_{\Theta} \mathbb{E}\left|R_{N}\left(\theta, X_{0}, X_{1}\right)\right|^{2} \mathbb{U}(d \theta) & =\sum_{N=1}^{\infty} \frac{1}{N} \sum_{n=1+N}^{\infty} \mathbb{E}\left|\mathcal{P}_{1} g\left(X_{n}\right)\right|^{2} \\
& =\sum_{N=1}^{\infty} \frac{1}{N}\left\|\mathbb{E}\left[g\left(X_{0}\right) \mid X_{-N}\right]\right\|^{2}<\infty .
\end{aligned}
$$

So for almost all $\theta \in \Theta(\mathbb{U})$,

$$
\sum_{N=1}^{\infty} \frac{1}{N} \mathbb{E}\left|R_{N}\left(\theta, X_{0}, X_{1}\right)\right|^{2}<\infty .
$$


It follows from Kronecker's lemma that as $N \rightarrow \infty$,

$$
\frac{1}{N} \sum_{j=1}^{N} \mathbb{E}\left|R_{j}\left(\theta, X_{0}, X_{1}\right)\right|^{2} \rightarrow 0,
$$

which yields

$$
\mathbb{E}\left|\frac{1}{N} \sum_{j=1}^{N} R_{j}\left(\theta, X_{0}, X_{1}\right)\right|^{2} \rightarrow 0
$$

by Cauchy's inequality. In other words,

$$
\bar{s}_{N}\left(\theta, X_{0}, X_{1}\right):=\frac{1}{N} \sum_{n=1}^{N} \mathcal{P}_{1} S_{n}(\theta) \rightarrow \xi\left(\theta, X_{0}, X_{1}\right) \quad \text { in } \mathcal{L}^{2} .
$$

Clearly, $\mathbb{E}\left[\bar{s}_{N}\left(\theta, X_{0}, X_{1}\right) \mid X_{0}\right]=0$ a.s. $(\mathbb{P})$. Hence as $N \rightarrow \infty$,

$$
\begin{aligned}
\mathbb{E}\left|\mathbb{E}\left[\xi\left(\theta, X_{0}, X_{1}\right) \mid X_{0}\right]\right| & =\mathbb{E}\left|\mathbb{E}\left[\xi\left(\theta, X_{0}, X_{1}\right)-\bar{s}_{N}\left(\theta, X_{0}, X_{1}\right) \mid X_{0}\right]\right| \\
& \leq \mathbb{E}\left|\xi\left(\theta, X_{0}, X_{1}\right)-\bar{s}_{N}\left(\theta, X_{0}, X_{1}\right)\right| \\
& \leq\left\|\xi\left(\theta, X_{0}, X_{1}\right)-\bar{s}_{N}\left(\theta, X_{0}, X_{1}\right)\right\| \rightarrow 0,
\end{aligned}
$$

which proves (14).

Lemma 4. Suppose (15) holds for $\theta$ for which $\xi\left(\theta, X_{0}, X_{1}\right) \in \mathcal{L}^{2}(\mathbb{P})$. Then

$$
\left(\begin{array}{c}
\Re \\
\Im
\end{array}\right) \frac{S_{n}(\theta)-\mathbb{E}\left[S_{n}(\theta) \mid X_{0}\right]}{\sqrt{n}} \Rightarrow N[0, \Sigma(\theta)],
$$

where $\Sigma(\theta)=\frac{1}{2}\left\|\xi\left(\theta, X_{0}, X_{1}\right)\right\|^{2} \operatorname{Id}_{2}$ if $\theta \neq 0, \pi$, and

$$
\Sigma(\theta)=\left(\begin{array}{cc}
\left\|\xi\left(\theta, X_{0}, X_{1}\right)\right\|^{2} & 0 \\
0 & 0
\end{array}\right), \quad \text { if } \theta=0, \pi .
$$

Proof of Lemma 4. We generalize the arguments in Woodroofe [21] where the asymptotic normality for the case $\theta=0$ was obtained. Let $\xi_{n}\left(\theta, x_{0}, x_{1}\right)=\mathbb{E}\left[S_{n}(\theta) \mid\right.$ $\left.X_{1}=x_{1}\right]-\mathbb{E}\left[S_{n}(\theta) \mid X_{0}=x_{0}\right]$, and write

$$
\begin{aligned}
S_{n}(\theta)-\mathbb{E}\left[S_{n}(\theta) \mid X_{0}\right] & =\sum_{j=1}^{n} e^{i \theta(j-1)} \xi\left(\theta, X_{j-1}, X_{j}\right) \\
& +\sum_{j=1}^{n} e^{i \theta(j-1)}\left[\xi_{n-j+1}\left(\theta, X_{j-1}, X_{j}\right)-\xi\left(\theta, X_{j-1}, X_{j}\right)\right] .
\end{aligned}
$$

Now we claim that (15) implies that the second part in the preceding display is $o_{\mathbb{P}}(\sqrt{n})$. Actually, by Lemma $3, \mathbb{E}\left[\xi_{n-j+1}\left(\theta, X_{j-1}, X_{j}\right)-\xi\left(\theta, X_{j-1}, X_{j}\right) \mid X_{j-1}\right]=0$ almost surely $\mathbb{P}$. Then it suffices to establish

$$
\frac{1}{n} \sum_{j=1}^{n}\left\|\xi_{n-j+1}\left(\theta, X_{j-1}, X_{j}\right)-\xi\left(\theta, X_{j-1}, X_{j}\right)\right\|^{2} \rightarrow 0,
$$

which follows from (15) via the stationarity of $X_{j}$. By Lemmaß3, $\xi\left(\theta, X_{j-1}, X_{j}\right), j \in$ $\mathbb{Z}$ is a martingale difference sequence. Hence we can use the martingale central limit theorem to deduce (16) from (17). To this end, let

$$
\begin{gathered}
\xi\left(\theta, X_{j-1}, X_{j}\right)=A_{j}(\theta)+i B_{j}(\theta), \\
\alpha_{j}(\theta)=A_{j}(\theta) \cos [(j-1) \theta]-B_{j}(\theta) \sin [(j-1) \theta], \\
\beta_{j}(\theta)=B_{j}(\theta) \cos [(j-1) \theta]+A_{j}(\theta) \sin [(j-1) \theta] .
\end{gathered}
$$


Then $A_{j}(\theta), B_{j}(\theta), \alpha_{j}(\theta)$ and $\beta_{j}(\theta)$ form martingale difference sequences, and

$$
\sum_{j=1}^{n} e^{i \theta(j-1)} \xi\left(\theta, X_{j-1}, X_{j}\right)=\sum_{j=1}^{n}\left[\alpha_{j}(\theta)+i \beta_{j}(\theta)\right] .
$$

For a fixed $0 \leq \theta_{0}<2 \pi$, let $D_{j}=\alpha_{j}(\theta) \cos \theta_{0}+\beta_{j}(\theta) \sin \theta_{0}$. Then the $D_{j}$ are again martingale differences. By the Cramer-Wold device, it suffices to show that

$\frac{1}{\sqrt{n}} \sum_{j=1}^{n} D_{j} \Rightarrow N\left[0, \sigma^{2}\left(\theta, \theta_{0}\right)\right], \quad$ where $\sigma^{2}\left(\theta, \theta_{0}\right)=\left(\cos \theta_{0}, \sin \theta_{0}\right) \Sigma(\theta)\left(\begin{array}{c}\cos \theta_{0} \\ \sin \theta_{0}\end{array}\right)$.

We now apply the martingale central limit theorem to $\sum_{j=1}^{n} D_{j} / \sqrt{n}$. The Lindeberg condition is automatically satisfied. Since $\mathbb{E}\left[\xi^{2}\left(\theta, X_{j-1}, X_{j}\right) \mid \mathcal{F}_{j-1}\right], j \in \mathbb{Z}$ forms a stationary and ergodic sequence in $\mathcal{L}^{1}(\mathbb{P})$, by Lemma 5 , the limit

$$
V(\theta)=\lim _{n \rightarrow \infty} \frac{1}{n} \sum_{j=1}^{n} \mathbb{E}\left[\xi^{2}\left(\theta, X_{j-1}, X_{j}\right) \mid \mathcal{F}_{j-1}\right] e^{2(j-1) i \theta}
$$

exists and equals 0 almost surely $(\mathbb{P})$ if $\theta \neq 0, \pi$, and equals $\left\|\xi\left(\theta, X_{0}, X_{1}\right)\right\|^{2}$ if $\theta=0$ or $\pi$. After some algebra,

$$
\begin{aligned}
& \Sigma_{11}(\theta)=\lim _{n \rightarrow \infty} \frac{1}{n} \sum_{j=1}^{n} \mathbb{E}\left[\alpha_{j}^{2}(\theta) \mid \mathcal{F}_{j-1}\right]=\frac{\left\|\xi\left(\theta, X_{0}, X_{1}\right)\right\|^{2}}{2}+\frac{\Re V(\theta)}{2} \\
& \Sigma_{22}(\theta)=\lim _{n \rightarrow \infty} \frac{1}{n} \sum_{j=1}^{n} \mathbb{E}\left[\beta_{j}^{2}(\theta) \mid \mathcal{F}_{j-1}\right]=\frac{\left\|\xi\left(\theta, X_{0}, X_{1}\right)\right\|^{2}}{2}-\frac{\Re V(\theta)}{2} \\
& \Sigma_{12}(\theta)=\lim _{n \rightarrow \infty} \frac{1}{n} \sum_{j=1}^{n} \mathbb{E}\left[\alpha_{j}(\theta) \beta_{j}(\theta) \mid \mathcal{F}_{j-1}\right]=\frac{\Im V(\theta)}{2}
\end{aligned}
$$

almost surely $(\mathbb{P})$, which completes the proof.

Lemma 5. Let $\eta_{n}, n \in \mathbb{Z}$, be a real-valued, stationary and ergodic sequence with $\mathbb{E}\left|\eta_{1}\right|<\infty$. Then for all $0<\alpha<2 \pi$,

$$
\frac{1}{n} \sum_{t=1}^{n} e^{i t \alpha} \eta_{t} \rightarrow 0
$$

almost surely and in $\mathcal{L}^{1}$.

Proof of Lemma 5. This result seems to be implied but not explicitly stated in the literature (see, for example, Doob (7], pp. 469-470), Rozanov (15], pp. 160161), Wiener and Wintner [20]). Since $\sum_{t=1}^{n} e^{i t \alpha} \mathbb{E}\left(\eta_{t}\right) / n \rightarrow 0$, we can assume, without loss of generality, that $\mathbb{E}\left(\eta_{t}\right)=0$. If $\alpha /(2 \pi)$ is a rational number, then 20) clearly follows from the classical ergodic theorem. In the case that $\alpha /(2 \pi)$ is not a rational number, define the rotation $\rho_{\alpha}: \Theta \mapsto \Theta$ by $\rho_{\alpha}(\theta)=\theta+\alpha \bmod 2 \pi$. Then $\rho_{\alpha}$ is measure-preserving and ergodic. Let the random variable $\vartheta \sim \operatorname{Uniform}(\Theta)$ be independent of $\eta_{n}, n \in \mathbb{Z}$. Since $\eta_{t}$ is stationary and ergodic, the sequence $\varphi_{t}=e^{i t \alpha+\vartheta} \eta_{t}, t \in \mathbb{Z}$, is then stationary (cf. Rozanov [15], p. 161) as well as ergodic on the product space $(\Theta \times \Omega, \mathcal{B}(\Theta) \times \mathcal{B}(\Omega), \mathbb{U} \times \mathbb{P})$. Therefore the classical ergodic theorem asserts that $n^{-1} \sum_{t=1}^{n} \varphi_{t} \rightarrow 0$ almost surely $(\mathbb{U} \times \mathbb{P})$ and in $\mathcal{L}^{1}$, which clearly entails (20) since $\mathbb{U}$ is a uniform distribution. 
Proof of Theorem 1. (i) This follows from Lemmas 1 and 4. (ii) By Lemmas 13 and 4 , for almost all $\theta, \varphi \in \Theta(\mathbb{U})$, martingale differences $\xi\left(\tau, X_{t-1}, X_{t}\right)=A_{t}(\tau)+$ $i B_{t}(\tau), \tau=\theta, \varphi$, can be constructed, and it suffices to show that $n^{-1 / 2} \sum_{t=1}^{n} \mathbf{v}_{t} \Rightarrow$ $N[0, \Sigma(\theta, \varphi)]$, where random vectors $\mathbf{v}_{t}=\left(\alpha_{t}(\theta), \beta_{t}(\theta), \alpha_{t}(\varphi), \beta_{t}(\varphi)\right)$ and the covariance matrix $\Sigma(\theta, \varphi)=\frac{1}{2} \operatorname{Diag}(w(\theta), w(\theta), w(\varphi), w(\varphi)), w(\tau)=\left\|\xi\left(\tau, X_{0}, X_{1}\right)\right\|^{2}$. To this end, by (i) and the Cramer-Wold device, (ii) follows from

$$
\frac{1}{n} \sum_{t=1}^{n}\left(\begin{array}{cc}
\alpha_{t}(\theta) \alpha_{t}(\varphi) & \alpha_{t}(\theta) \beta_{t}(\varphi) \\
\beta_{t}(\theta) \alpha_{t}(\varphi) & \beta_{t}(\theta) \beta_{t}(\varphi)
\end{array}\right) \rightarrow 0 \quad \text { a.s. } \quad(\mathbb{P}),
$$

which in view of Lemma 5 is valid provided that $\varphi \neq \theta$ and $\varphi+\theta \neq 2 \pi$.

Proof of Theorem 2. Observe that (16) in Lemma 4 holds for $\theta$ for which (4) is satisfied. Therefore, (6) follows in view of (5).

Proof of Proposition [1. Let $h_{n}=\sum_{t=1}^{n} e^{i t \theta}$. Since $0<\theta<2 \pi, h:=\sup _{n \geq 1}\left|h_{n}\right|<$ $\infty$. By (7), $\sum_{n=1}^{\infty} h_{n}\left[\mathcal{P}_{1} g\left(X_{n}\right)-\mathcal{P}_{1} g\left(X_{n+1}\right)\right]$ converges in $\mathcal{L}^{2}(\mathbb{P})$. Thus (4) holds since $\left\|\mathcal{P}_{1} g\left(X_{n}\right)\right\| \rightarrow 0$. To prove (5), let $\phi_{t}=\left\|\mathcal{P}_{1} g\left(X_{t}\right)-\mathcal{P}_{1} g\left(X_{t+1}\right)\right\|$ and $\Phi_{k}=$ $\sum_{t=k}^{\infty} \phi_{t}$. Then (7) entails $\sum_{j=0}^{\infty}\left[\sum_{t=1}^{n} \phi_{t+j}\right]^{2} \leq \Phi_{1} \sum_{j=0}^{\infty} \sum_{t=1}^{n} \phi_{t+j}=o(n)$. So (5) follows from

$$
\left\|\mathcal{P}_{-j} S_{n}(\theta)\right\| \leq h \sum_{t=1}^{n}\left\|\mathcal{P}_{-j} g\left(X_{t}\right)-\mathcal{P}_{-j} g\left(X_{t+1}\right)\right\|+h\left\|\mathcal{P}_{-j} g\left(X_{n+1}\right)\right\|,
$$

$\left\|\mathbb{E}\left[S_{n}(\theta) \mid X_{0}\right]\right\|^{2}=\sum_{j=0}^{\infty}\left\|\mathcal{P}_{-j} S_{n}(\theta)\right\|^{2}$ and $\sum_{j=0}^{\infty}\left\|\mathcal{P}_{-j} g\left(X_{n+1}\right)\right\|^{2} \leq\left\|g\left(X_{0}\right)\right\|^{2}$ by the orthogonality of $\mathcal{P}_{k}$.

Proof of Proposition Q Let $K_{n}(\lambda)=\sin ^{2}(n \lambda / 2) /\left[2 \pi n \sin ^{2}(\lambda / 2)\right]$ be the Fejér kernel. It is well known that $\left\|S_{n}(\theta)\right\|^{2} /(2 \pi n)=\int_{0}^{2 \pi} K_{n}(\lambda-\theta) f(\lambda) d \lambda$ (cf. Theorem 8.2.7 in Anderson ([1], p. 454)), which by the Fejér-Lebesgue Theorem (cf. Bary (2], p. 139)) converges almost everywhere to $f(\theta)$. Therefore, by (17) and (18), for almost all $\theta$ (Lebesgue),

$$
\lim _{n \rightarrow \infty} \frac{1}{n}\left\|\mathbb{E}\left[S_{n}(\theta) \mid X_{0}\right]\right\|^{2}=\lim _{n \rightarrow \infty} \frac{1}{n}\left\|S_{n}(\theta)\right\|^{2}-\lim _{n \rightarrow \infty} \frac{1}{n}\left\|S_{n}(\theta)-\mathbb{E}\left[S_{n}(\theta) \mid X_{0}\right]\right\|^{2}
$$

Notice that by (8),

$$
\sum_{k=1}^{\infty} 2^{-k} \int_{0}^{2 \pi}\left\|\mathbb{E}\left[S_{2^{k}}(\theta) \mid X_{0}\right]\right\|^{2} \mathbb{U}(d \theta)=\sum_{k=1}^{\infty} 2^{-k} \sum_{i=1}^{2^{k}}\left\|\mathbb{E}\left[g\left(X_{i}\right) \mid X_{0}\right]\right\|^{2}<\infty .
$$

Hence by Borel-Cantelli's Lemma, for almost all $\theta$ (Lebesgue), $2^{-k}\left\|\mathbb{E}\left[S_{2^{k}}(\theta) \mid X_{0}\right]\right\|^{2}$ $\rightarrow 0$ as $k \rightarrow \infty$, which by (21) entails $2 \pi f(\theta)=\left\|\xi\left(\theta, X_{0}, X_{1}\right)\right\|^{2}=2 \sigma^{2}(\theta)$ almost surely.

\section{ACKNOWLEDGMent}

The author is grateful to the referee for many helpful suggestions. 


\section{REFERENCES}

1. Anderson, T. W. (1971). The Statistical Analysis of Time Series. Wiley, New York. MR 44:1169

2. Bary, N. K. (1964). A Treatise on Trigonometric Series. Macmillan, New York. MR30:1347

3. Brockwell, P. J. and Davis, R. A. (1991). Time Series: Theory and Methods. Springer, New York. MR92d:62001

4. Carleson, L. (1966). On convergence and growth of partial sums of Fourier series. Acta Math. 116 135-157. MR 33:7774

5. Chow, Y. S. and Teicher, H. (1988). Probability Theory. 2nd ed. New York, Springer. MR89e:60001

6. Diaconis, P. and Freedman. D. (1999). Iterated random functions. SIAM Rev. $4141-76$. MR2000c:60102

7. Doob, J. (1953). Stochastic Processes. Wiley, New York. MR15:445b

8. Gray, H. L., Zhang, N.-F. and Woodward, W.A. (1989). On generalized fractional processes. J. Time Ser. Anal. 10 233-257. MR90m:62208

9. Ibragimov, I. A. and Linnik, Yu. V. (1971). Independent and stationary sequences of random variables. Wolters-Noordhoff, Groningen. MR 48:1287

10. Meyn, S. P. and Tweedie, R. L. (1993). Markov chains and stochastic stability. Springer, London; New York. MR95j:60103

11. Olshen, R. A. (1967). Asymptotic properties of the periodogram of a discrete stationary process. J. Appl. Probab. 4 508-528. MR37:3643

12. Rootzén, H. (1976). Gordin's theorem and the periodogram. J. Appl. Probab. 13 365-370. MR53:14619

13. Rosenblatt, M. (1981). Limit theorems for Fourier transforms of functionals of Gaussian sequences. Z. Wahrsch. Verw. Gebiete 55 123-132. MF,82i:60066

14. Rosenblatt, M. (1985). Stationary sequences and random fields. Birkhäuser, Boston. MR 88c:60077

15. Rozanov, Yu. A. (1967). Stationary random processes. Holden-Day, San Francisco. MR 35:4985

16. Terrin, N. and Hurvich, C. M. (1994). An asymptotic Wiener-Ito representation for the low frequency ordinates of the periodogram of a long memory time series. Stochastic Process. Appl. 54 297-307. MR95i:62078

17. Tong, H. (1990). Non-linear time series: a dynamical system approach. Oxford University Press. MF 92a:62141

18. Walker, A. M. (1965). Some asymptotic results for the periodogram of a stationary time series. J. Austral. Math. Soc. 5 107-128. MR.31:1720

19. Walker, A. M. (2000). Some results concerning the asymptotic distribution of sample Fourier transforms and periodograms for a discrete-time stationary process with a continuous spectrum. J. Time Ser. Anal. 21 95-109. MR2001c:62108

20. Wiener, N. and Wintner, A. (1941). Harmonic analysis and ergodic theory. American Journal of Mathematics 63 415-426. MF 2:319b

21. Woodroofe, M. (1992). A central limit theorem for functions of a Markov chain with applications to shifts. Stochastic Process. Appl. 41 33-44. MR 93d:60037

22. Wu, W. B. and Woodroofe, M. (2000). A central limit theorem for iterated random functions. J. Appl. Probab. 37 748-755. MR2002d:60020

23. Yajima, Y. (1989). A central limit theorem of Fourier transforms of strongly dependent stationary processes. J. Time Ser. Anal. 10 375-383. MR 91a:62258

Department of Statistics, The University of Chicago, 5734 S. University Avenue, Chicago, Illinois 60637

E-mail address: wbwu@galton.uchicago.edu 